\title{
Eating 'attentively' reduces later energy consumption in overweight and obese females
}

\author{
Eric Robinson $^{1 *}$, Inge Kersbergen ${ }^{1}$ and Suzanne Higgs ${ }^{2}$ \\ ${ }^{1}$ Eleanor Rathbone Building, University of Liverpool, Liverpool L69 7ZA, UK \\ ${ }^{2}$ University of Birmingham, Edgbaston, Birmingham B15 2TT, UK \\ (Submitted 6 February 2014 - Final revision received 28 April 2014 - Accepted 12 May 2014 - First published online 16 June 2014)
}

\section{Abstract}

Attentional and memory processes underpin appetite control, but whether encouraging overweight individuals to eat more 'attentively' can promote reductions in energy consumption is unclear. In the present study with a between-subjects design, a total of forty-eight overweight and obese females consumed a fixed lunchtime meal. Their ad libitum energy intake of high-energy snack food was observed during a second laboratory session that occurred later that day. In the focused-attention condition, participants ate their lunch while listening to audio instructions that encouraged them to pay attention to the food being eaten. In a control condition, participants ate while listening to an audio book with a neutral (non-food-related) content. To test whether focused attention influenced food intake via enhancing the memory of the earlier consumed meal, we measured the participants' memory of their lunchtime meal. Ad libitum snack intake was approximately $30 \%$ lower for participants in the focused-attention condition than for those in the control condition, and this difference was statistically significant. There was limited evidence that attention decreased later food intake by enhancing memory representation of the earlier consumed meal. Eating attentively can lead to a substantial decrease in later energy intake in overweight and obese individuals. Behavioural strategies that encourage a more 'attentive' way of eating could promote sustained reductions in energy intake and weight loss.

\section{Key words: Attentive eating: Appetite: Focused attention: Food memory}

Appetite is governed by complex interactions between multiple regulatory factors, including cognition ${ }^{(1-3)}$. Identifying key cognitive drivers of appetite and energy consumption is likely to be important, as it may enable us to develop more effective interventions to help overweight and obese individuals make sustained reductions in their energy consumption and support weight loss ${ }^{(4)}$

We recently meta-analysed studies that have examined the role of attention and memory in energy consumption ${ }^{(5)}$. The results of these studies indicated that eating while distracted increases energy intake ${ }^{(6)}$ and that enhancing and impairing episodic memory for recent eating experiences is associated with decreases and increases in later food intake, respectively ${ }^{(7,8)}$. We interpreted these findings using an episodic memory account, whereby memory informs appetite regulation by providing information about the satiating effects of recent eating experiences ${ }^{(9,10)}$. Based on this framework, we proposed that eating more 'attentively' (by focusing attention towards the food being consumed) could be a way of promoting reductions in energy consumption. Higgs \& Donohoe ${ }^{(7)}$ tested this proposition in healthy-weight female college students and found that focusing attention during a lunchtime meal reduced later food consumption, and this reduction in food intake was associated with enhanced memory for the earlier consumed meal.

Although mindfulness techniques or encouraging a more 'mindful' approach to eating behaviour has received some attention $^{(11)}$ and behavioural monitoring can help individuals make changes to their dietary behaviour and lose weight ${ }^{(12)}$, these techniques are distinct to 'attentive' eating, as they do not target appetite through memory or attentional processes. Instead, they encourage individuals to experience difficult thoughts or feelings rather than trying to avoid them ${ }^{(11)}$ and prompt them to monitor goal progress ${ }^{(12)}$. Although encouraging a more attentive way of eating could be an effective way of helping people reduce their energy intake and lose weight ${ }^{(13)}$, we are not aware of any experimental studies that have examined whether this could be an effective approach for overweight and obese individuals to adopt. Given that increasing attention to food being consumed as it is eaten has been shown to decrease later food intake among healthy-weight participants ${ }^{(7)}$, the primary aim of the present study was to test whether increasing attention to food being consumed ('attentive eating') leads to reductions in later energy consumption in overweight and obese participants. 


\section{Methods}

\section{Participants}

A total of forty-eight overweight and obese females, with a mean age of 32.6 (SD 13.5) years, participated in the present study. The study was advertised as examining 'taste and food preference' and participants were recruited from a UK university campus and the surrounding community. Participants who registered interest in the study were only eligible for participation if they were female, aged 18 years or older, fluent speakers of English, were not taking medication that affected their appetite, had no known history of food allergies and had a self-reported BMI $\geq 25 \mathrm{~kg} / \mathrm{m}^{2}$. Based on the large effect size that Higgs \& Donohoe ${ }^{(7)}$ observed (standardised mean difference $0 \cdot 88$ ), a power calculation (75\% power, G*Power 3.1; http://www.softpedia.com/get/Science-CAD/G-Power. shtml) indicated that we would require a sample size of approximately forty-six participants. We recruited slightly above this number in case any participants withdrew from the study. All participants completed the study. The present study was conducted according to the guidelines laid down in the Declaration of Helsinki, and all procedures were approved by the University of Liverpool Research Ethics Committee. Written informed consent was obtained from all participants.

\section{Design}

Participants were randomly assigned to one of two conditions. In the focused-attention condition, participants listened to verbal instructions that encouraged them to pay attention to the food they were eating. They were instructed to focus on and pay attention to the look, smell and flavour of the food $^{(7)}$. In the control condition, participants listened to a fragment of a neutral audio book about the cuckoo bird. The piece describes the arrival of the cuckoo in Britain, the upbringing of their young and their notable cry. Both audio clips lasted $3 \mathrm{~min}$. We opted for a between-subjects design in the present study, as use of a repeated-measures design for a study of this nature would heighten demand awareness and make the aims apparent to the participants ${ }^{(8)}$.

\section{Test foods}

Participants were offered a ham sandwich and a portion of crisps for lunch. Vegetarians ate a cheese sandwich instead of ham. There were two vegetarians in the control condition and one vegetarian in the focused-attention condition. The ham sandwich was $879 \mathrm{~kJ} / 100 \mathrm{~g}$ (210 kcal/100 g), comprising $1004 \mathrm{~kJ}$ ( $240 \mathrm{kcal}), 5 \mathrm{~g}$ fat (2 $\mathrm{g}$ saturated), $34 \mathrm{~g}$ carbohydrates (3g sugars) and $14 \mathrm{~g}$ protein per portion. The cheese sandwich was $1172 \mathrm{~kJ} / 100 \mathrm{~g}$ ( $280 \mathrm{kcal} / 100 \mathrm{~g})$, comprising $1611 \mathrm{~kJ}$ (385 kcal), $18 \mathrm{~g}$ fat (10 g saturated), $27 \mathrm{~g}$ carbohydrates $(2 \mathrm{~g}$ sugars) and $13 \mathrm{~g}$ protein per portion. The crisps were $2201 \mathrm{~kJ} / 100 \mathrm{~g}$ (526 kcal/100 g), comprising $715 \mathrm{~kJ}$ (171 kcal), $10 \mathrm{~g}$ fat ( $1 \mathrm{~g}$ saturated), $17 \mathrm{~g}$ carbohydrates ( $0 \mathrm{~g}$ sugars) and $2 \mathrm{~g}$ protein per portion. For the afternoon ad libitum snack, participants were provided with well-stocked bowls of three types of cookie, broken into quarters; chocolate fingers
(2176 kJ/100 g (520 kcal/100 g)), digestive biscuits (2071 kJ/100 g $(495 \mathrm{kcal} / 100 \mathrm{~g})$ ) and chocolate-chip cookies $(2138 \mathrm{~kJ} / 100 \mathrm{~g}$ $(511 \mathrm{kcal} / 100 \mathrm{~g})$ ). Foods were purchased from Tesco Supermarket, UK.

\section{Procedure}

After being screened for eligibility, participants attended the first test session that took place between 12.00 and 14.00 hours. Participants first completed a medical history questionnaire to ensure they did not have any food-related allergies. Participants next completed a set of $100 \mathrm{~mm}$ paper-based visual analogue scales (anchors: 'not at all' to 'extremely') to measure hunger (e.g. 'how hungry do you feel right now?') and various mood dimensions. Next, the researcher brought the lunchtime meal with a glass of water and provided the participants with headphones. Participants were instructed to listen carefully to the audio clip and were told that they would have approximately $10 \mathrm{~min}$ to consume their lunch and that they should try to finish all of the meal. After $10 \mathrm{~min}$, the researcher returned and provided the participants with the same set of appetite and mood rating scales to complete. To corroborate the cover story, participants also completed a questionnaire that asked them to rate how the meal tasted (e.g. 'how salty was the meal?'). We did not specifically draw attention to the purpose of the audio clips, but in line with the cover story of 'taste perception', we reasoned that participants may believe that the study examined how an audio clip influenced taste perception.

At $2-3 \mathrm{~h}$ later, participants returned for the second test session. They completed the same appetite and mood rating scales, before being provided with the snacks. Participants were left for $10 \mathrm{~min}$ and provided with another taste rating questionnaire to complete and were told that they could eat as much as they liked once they were finished. Participants then completed the appetite and mood rating scales again. Next, they were asked to rate how vivid their memory of their lunchtime meal was (100 mm paper-based visual analogue scale, with anchors ranging from 'not at all' to 'extremely'), as well as were asked how many calories they thought they had eaten at lunch (no guidance or instructions were provided). Participants next completed the Dutch Eating Behaviour Questionnaire ${ }^{(14)}$ and demographic questions, and wrote down what they thought the study was about. Participants were then weighed and had their height measured to calculate BMI and were debriefed. A non-blinded researcher scored all ratings made on the visual analogue scales.

\section{Statistical analysis}

We compared the conditions on all outcome measures using independent-sample $t$ tests as data were normally distributed. To examine whether ad libitum consumption was associated with meal memory, we used Pearson's correlation coefficient $(r)$. 
Table 1. Characteristics of the study participants as a function of condition (Mean values and standard deviations)

\begin{tabular}{|c|c|c|c|c|c|c|}
\hline & \multicolumn{2}{|c|}{$\begin{array}{l}\text { Focused-attention } \\
\text { condition ( } n 25)\end{array}$} & \multicolumn{2}{|c|}{$\begin{array}{l}\text { Control condition } \\
\qquad(n 23)\end{array}$} & \multirow[b]{2}{*}{$t$ test $^{*}$} & \multirow[b]{2}{*}{$P^{\star}$} \\
\hline & Mean & SD & Mean & SD & & \\
\hline Age (years) & $31 \cdot 1$ & $12 \cdot 3$ & $34 \cdot 1$ & 14.7 & 0.77 & 0.44 \\
\hline $\mathrm{BMI}\left(\mathrm{kg} / \mathrm{m}^{2}\right)$ & 30.3 & 3.9 & $28 \cdot 3$ & 3.7 & 1.82 & 0.08 \\
\hline DEBQ restraint scale $\dagger$ & $30 \cdot 6$ & $10 \cdot 2$ & $30 \cdot 1$ & $7 \cdot 3$ & $0 \cdot 17$ & 0.87 \\
\hline DEBQ external eating scale $†$ & $32 \cdot 4$ & 4.9 & 39.9 & 24.4 & 1.51 & 0.14 \\
\hline DEBQ emotional eating scale & $38 \cdot 7$ & $13 \cdot 2$ & $38 \cdot 7$ & $10 \cdot 8$ & $0 \cdot 12$ & 0.99 \\
\hline
\end{tabular}

DEBQ, Dutch Eating Behaviour Questionnaire.

${ }^{*} \mathrm{df}=46$.

†Scale scores are summed averages.

\section{Results}

\section{Participants}

The mean BMI measured on the day of testing was $29 \cdot 3$ (sD 3.4$) \mathrm{kg} / \mathrm{m}^{2}$. The two groups did not differ significantly in age, BMI, eating behaviour traits or baseline hunger (see Tables 1 and 2), indicating that randomisation was successful. Only five participants did not consume all of the lunchtime meal (four from the focused-attention condition and one from the control condition). Controlling for energy consumed at lunch did not influence the significant and non-significant patterns of any of the observed results relating to later ad libitum intake or hunger.

\section{Ad libitum snack intake}

Participants in the focused-attention condition ate significantly less than those in the control condition during the afternoon session $(t(46)=2 \cdot 5, P=0.015)$. The mean consumption of participants in the focused-attention condition was 1046 (SD $385) \mathrm{kJ}$ (250 (SD 92) kcal) and that of participants in the control condition was 1490 (SD 774) kJ (356 (SD 185) kcal), which constitutes a $30 \%$ reduction in energy consumption.

We also examined whether the effect of condition on intake differed according to the three different types of cookie used. Entering condition (focused attention $v$. control) as a betweensubject factor and cookie type (chocolate chip, chocolate fingers and digestive biscuits) as a within-subject factor into ANOVA, the interaction between condition and cookie type was not found to be statistically significant $(F=1 \cdot 851$,
$P=0 \cdot 16$ ), suggesting that participants ate less in general and the specific type of cookie used did not significantly moderate whether an effect of condition on intake was observed. The mean intake of each cookie type for the two conditions was as follows: chocolate-chip cookies - focused attention $14 \mathrm{~g}$ and control condition $23 \mathrm{~g}$; chocolate fingers - focused attention $24 \mathrm{~g}$ and control condition $33 \mathrm{~g}$; digestive biscuits focused attention $11 \mathrm{~g}$ and control condition $13 \mathrm{~g}$.

\section{Hunger}

Although participants in the focused-attention condition ate significantly less than those in the control condition during the ad libitum intake session, the two groups reported similar post-consumption hunger levels $(P=0 \cdot 90)$. There was no evidence of between-condition differences for self-reported hunger at any of the other time points during the study (see Table 2).

\section{Memory measures}

Participants in both conditions had similarly vivid memories of their lunchtime meal $(t(46)=0 \cdot 9, P=0 \cdot 86$; focused-attention condition 83.0 (SD 13.3) and control condition 83.7 (SD 9.7)) We measured the participants' memory accuracy for the amount of food they had eaten by subtracting the actual lunch energy content from later estimated consumption (how close the participants were to the exact value). Both conditions overestimated how much they had eaten and the focused-attention condition $(380.7$ (SD 679.5) kJ (91.0

Table 2. Self-reported hunger* by condition (Mean values and standard deviations)

\begin{tabular}{|c|c|c|c|c|c|c|}
\hline & \multicolumn{2}{|c|}{$\begin{array}{l}\text { Focused-attention } \\
\text { condition ( } n 25)\end{array}$} & \multicolumn{2}{|c|}{$\begin{array}{c}\text { Control condition } \\
(n 23)\end{array}$} & \multirow[b]{2}{*}{$t$ test† } & \multirow[b]{2}{*}{$P \dagger$} \\
\hline & Mean & SD & Mean & SD & & \\
\hline Pre-lunch hunger & $57 \cdot 2$ & $23 \cdot 2$ & $56 \cdot 8$ & 21.5 & 0.06 & 0.95 \\
\hline Post-lunch hunger & 13.4 & $14 \cdot 0$ & 20.9 & 18.5 & 1.60 & 0.12 \\
\hline Pre-ad libitum snack hunger & $35 \cdot 8$ & $23 \cdot 0$ & $42 \cdot 7$ & $27 \cdot 1$ & 0.95 & 0.35 \\
\hline Post-ad libitum snack hunger & 18.6 & 21.9 & $17 \cdot 9$ & $16 \cdot 1$ & 0.12 & 0.90 \\
\hline
\end{tabular}

*Hunger measured using a $100 \mathrm{~cm}$ visual analogue scale, with anchors ranging from 'not at all' to 'extremely'. $\dagger \mathrm{df}=46$. 
(sD 162.4) kcal)) tended to be more accurate than the control condition (648.9 (SD 630.1) kJ (155.1 (SD 150.6) kcal)), although this difference was not statistically significant $(t(46)=1 \cdot 4, P=0 \cdot 16)$. Neither memory vividness $(r-0 \cdot 12$, $P=0 \cdot 42)$, nor accuracy for the amount of food eaten $(r 0.06$, $P=0.67)$ was associated with energy consumption.

\section{Sensitivity analysis}

Removal of outliers. In addition to the main analyses described above, we also examined the effect of removing outlying data points on our planned analyses. There was one outlier (defined as a data point of two or more standard deviations away from the mean) for the main dependent variable (ad libitum energy consumption). Removal of this data point did not influence the results. There was an outlier for the measure 'accuracy for the amount of food eaten' (overestimation of $2510 \cdot 4 \mathrm{~kJ}(600 \mathrm{kcal}))$. Removal of this data point resulted in the difference between the focused attention $(297 \cdot 1(\mathrm{SD} 548 \cdot 1) \mathrm{kJ}(71 \cdot 0(\mathrm{SD} 131 \cdot 0) \mathrm{kcal}))$ and (649.4 (SD $630 \cdot 1) \mathrm{kJ} \quad(155 \cdot 2 \quad(\mathrm{sD}$ 150.6) kcal)) becoming significant $(t(46)=2 \cdot 0, P=0 \cdot 047)$. Removal of outliers did not affect the relationships between ad libitum energy consumption and the two memory measures.

\section{Demand awareness and eligibility criteria}

None of the participants correctly identified the main aims of the present study. Of the forty-eight participants, sixteen mentioned that the amount of food they had eaten may have been assessed during the study, but including this as a factor in the analysis $(2 \times 2$ ANOVA: experimental condition by aware $v$. unaware) did not affect the effect that condition had on energy intake. The BMI of three participants just missed the overweight weight range (e.g. BMI of $24.6 \mathrm{~kg} / \mathrm{m}^{2}$ ) when their BMI was calculated on the day of testing. Removal of their data did not change the observed results.

\section{Discussion}

Overweight and obese participants who focused their attention on what they were eating for lunch ate significantly less during a laboratory snack-tasting session later that day than participants in a control condition. The size of this reduction in energy intake was approximately $30 \%$. Participants in both conditions had similarly vivid memories of their lunchtime meal, suggesting that differences in food memory may not explain the results, although there was some evidence that the focused-attention condition may have had more accurate memory representations of how much they had eaten at lunch. This is the first study to examine the effect that focused attention has on appetite regulation in overweight and obese participants, and our findings support the notion that behavioural strategies that encourage individuals to focus on and pay attention to their food and eat in a more 'attentive' manner may help to promote reductions in energy intake ${ }^{(4)}$.

Higgs \& Donohoe ${ }^{(7)}$ found that self-reported memory vividness was elevated in the focused-attention condition, and this measure was correlated with later energy intake. In the present study, scores for self-reported memory vividness were high across the two conditions, which may have constituted a ceiling effect ${ }^{(15)}$. Although there was some evidence that participants in the focused-attention condition were more accurate when estimating the amount of food they had consumed for lunch, this accuracy was not associated with later energy intake. It may be that if we had included a more specific measure of interoceptive memory, it would have explained the effect that attention had on consumption, as episodic memory may primarily govern appetite through providing information about the satiating effects of recent eating experiences $^{(16,17)}$.

The present study had some limitations. We only sampled females and observed ad libitum energy intake during a single laboratory session. Future work will benefit from examining this novel dietary approach over longer periods, in order to determine whether sustained reductions in energy intake are observed. Given that the decrease in food intake that we observed in the focused-attention condition was not associated with an elevation in post-consumption hunger levels, the longer-term effects of this approach now warrant investigation.

\section{Conclusions}

Focusing overweight and obese individuals' attention to food they are eating can lead to substantial reductions in later energy intake. A more 'attentive' approach to eating could promote sustained reductions in energy intake and weight loss.

\section{Acknowledgements}

There is no external funding to report.

The authors' contributions are as follows: E. R., S. H. and I. K. conceived the study, analysed the data and were involved in the writing of the manuscript; I. K. conducted the study.

None of the authors has any conflicts of interest to declare.

\section{References}

1. Speakman JR (2004) Obesity: the integrated roles of environment and genetics. J Nutr 134, 2090S-2105S.

2. Booth DA (2008) Physiological regulation through learnt control of appetites by contingencies among signals from external and internal environments. Appetite $\mathbf{5 1}$, 433-441.

3. Hetherington MM (2002) The physiological-psychological dichotomy in the study of food intake. Proc Nutr Soc 61, 497-507.

4. Higgs S, Robinson E \& Lee M (2012) Learning and memory processes and their role in eating: implications for limiting food intake in overeaters. Curr Obes Rep 1, 91-98.

5. Robinson E, Aveyard PN, Daley A, et al. (2013) Eating attentively: a systematic review and meta-analysis of the effect of food intake memory and awareness on eating. Am J Clin Nutr 97, 728-742. 
6. Blass EM, Anderson DR, Kirkorian HL, et al. (2006) On the road to obesity: television viewing increases intake of high-density foods. Physiol Behav 88, 597-604.

7. Higgs S \& Donohoe J (2011) Focusing on food during lunch enhances lunch memory and decreases later snack intake. Appetite 57, 202-206.

8. Oldham-Cooper RE, Hardman CA, Nicoll CE, et al. (2011) Playing a computer game during lunch affects fullness, memory for lunch, and later snack intake. Am J Clin Nutr 93, 308-313

9. Higgs S (2002) Memory for recent eating and its influence on subsequent food intake. Appetite 39, 159-166.

10. Higgs S (2008) Cognitive influences on food intake: the effects of manipulating memory for recent eating. Physiol Behav 94, 734-739.

11. Tapper K, Shaw C, Ilsey J, et al. (2009) Exploratory randomised controlled trial of a mindfulness-based weight loss intervention for women. Appetite 52, 396-404.
12. Baker RC \& Kirschenbaum DS (1993) Self-monitoring may be necessary for successful weight control. Behav Ther $\mathbf{2 4}$, 377-394

13. Robinson E, Higgs S, Daley AJ, et al. (2013) Developing and feasibility testing a smartphone based attentive eating intervention. BMC Public Health 13, 639.

14. Van Strien T, Frijters JER, Bergers GPA, et al. (1986) The Dutch Eating Behavior Questionnaire (DEBQ) for assessment of restrained, emotional, and external eating behavior. Int J Eat Disord 5, 295-315.

15. Higgs S \& Jones A (2013) Prolonged chewing at lunch decreases later snack intake. Appetite 62, 91-95.

16. Higgs S, Williamson AC \& Attwood AS (2008) Recall of recent lunch and its effect on subsequent snack intake. Physiol Behav 94, 454-462.

17. Brunstrom JM, Burn JF, Sell NR, et al. (2012) Episodic memory and appetite regulation in humans. PLOS ONE 7 , e50707. 